\title{
Density-functional calculations of magnetoplasmons in quantum rings
}

\author{
A. Emperador \\ Departament d'Estructura i Constituents de la Matèria, Facultat de Física, Universitat de Barcelona, E-08028 Barcelona, Spain \\ M. Barranco* and E. Lipparini \\ Dipartimento di Fisica, Università di Trento, and INFM sezione di Trento, I-38050 Povo, Italy \\ M. Pi \\ Departament d'Estructura i Constituents de la Matèria, Facultat de Física, Universitat de Barcelona, E-08028 Barcelona, Spain \\ L1. Serra \\ Departament de Física, Facultat de Ciències, Universitat de les Illes Balears, E-07071 Palma de Mallorca, Spain \\ (Received 2 December 1998)
}

\begin{abstract}
We have studied the structure and dipole charge-density response of nanorings as a function of the magnetic field using local-spin-density-functional theory. Two small rings consisting of 12 and 22 electrons confined by a positively charged background are used to represent the cases of narrow and wide rings. The results are qualitatively compared with experimental data existing on microrings and on antidots. A smaller ring containing five electrons is also analyzed to allow for a closer comparison with a recent experiment on a two-electron quantum ring. [S0163-1829(99)02723-X]
\end{abstract}

\section{INTRODUCTION}

The study of collective excitations in bounded twodimensional electron systems (2DES's) is a subject of current interest, especially for the particular geometry called "quantum dot" in which a number of electrons is confined into a rather small, almost two-dimensional region produced by present available etching technologies, and for the quasione-dimensional structures called quantum wires (see, for example, Refs. 1 and 2 for a comprehensive description of quantum dots and wires). Less effort has been put in the investigation of these excitations in quantum antidots, i.e., the reversed structure of dots made in the 2DES's. ${ }^{3-8}$

Recently, magnetoplasmons arising in a ring-confining geometry have also attracted some interest. The first experimental studies concerned structures on the micron scale, etched into a molecular-beam-epitaxy-grown $\delta$-doped GaAs$\mathrm{Ga}_{x} \mathrm{Al}_{1-x} \mathrm{As}$ heterostructure, of outer diameter $\sim 50 \mu \mathrm{m}$ and inner diameter in the $12-30 \mu \mathrm{m}$ range. The observed magnetoplasmon resonances ${ }^{9}$ bear some of the properties of the dynamical response of a classical 2DES. ${ }^{10}$ Later on, a hydrodynamic theory based on the Thomas-Fermi-Dirac-von Weizsäcker approximation has been used $^{11}$ to describe $N$ $=400$ electron rings which yields a good account of the experimental data after an appropriate scaling of them at zero magnetic field $(B)$. Plasmon modes in very narrow rings have been described within a Hartree plus random-phase approximation, ${ }^{12}$ and the charge-density response of a dot with a repulsive impurity in its center has also been worked out. ${ }^{13}$ The optical absorption and inelastic scattering of a two-electron quantum ring of a rather large radius $(480 \mathrm{~nm})$ and width $(20 \mathrm{~nm})$ has been discussed in detail, ${ }^{14}$ and singleelectron properties of quantum rings with parabolic confinement have been discussed, ${ }^{15}$ with the aim of determining the effect of electron-electron interactions on the energy spec- trum and magnetic moment associated with the persistent current in a quantum ring. ${ }^{16}$

The far-infrared (FIR) charge-density excitation (CDE) appears to depend on the ring width. The measured CDE's (Ref. 9) are bundled into a high-energy group and a lowenergy group, which in contradistinction with the case of dots do not merge at $B=0$. The low-energy peaks arrange into two distinct branches. For narrow rings (NR's), both have a negative $B$ dispersion, whereas for broad rings (BR's) one branch displays a positive $B$ dispersion at small magnetic fields. The high energy peaks arrange into one (narrow ring) or several (broad ring) branches. The high-energy branches display a negative $B$ dispersion at small magnetic fields.

The low-energy peaks have been explained as edge magnetoplasmons excited at the inner and outer boundaries of the ring, ${ }^{10,11}$ whereas the high-energy peaks are bulk magnetoplasmons. ${ }^{11}$ It is worth recalling that in the case of antidots, only one edge magnetoplasmon is detected whose energy goes to zero with $B .{ }^{3,4}$ It thus seems that the observed ring plasmons exhibit features of either dots or antidots depending on the ring wideness and $B$ value.

Very recently, nanorings in InAs-GaAs heterostructures have been fabricated in the $15-40 \mathrm{~nm}$ radius range, ${ }^{17}$ and the FIR response has been measured for a two-electron ring. ${ }^{18}$ Two sets of peaks appear in the response, as in the case of microrings. Depending on the $B$ value, $1-3$ main peaks have been detected and arranged into four energy branches with $B$ dispersions which seem to differ from the microring systematics. ${ }^{18}$ The two branches starting from the $B=0$ high-energy peak are similar to those of quantum dots, and according to the analysis of the experimental data presented in Ref. 18, the two branches corresponding to the low-energy peaks both seem to display a positive $B$ dispersion. It is worth noting that the experimental results on microrings cover a low- $B$ range (up to $2 \mathrm{~T}$ ), whereas the ones on nan- 
orings extend up to $14 \mathrm{~T}$, but no data on the low-energy nanoring peaks have been recorded below $4 \mathrm{~T}$.

The studied nanorings present an elongation in the $[1,-1,0]$ direction. It is likely not preventing the electrons too much from being circularly distributed. Otherwise, at $B$ $=0$ the two high-energy branches would not merge at all, as they seem to do. A similar situation, namely, a noncircularly symmetric dot hosting a quite circularly symmetric electronic density, is also found for few-electron quantum dots. ${ }^{19}$ In addition, during their manufacture nanorings had to be further covered to complete the necessary layer structure. ${ }^{18}$ All this might result in nontrivial changes with respect to the CDE's of a "clean," circularly symmetric ring, and it calls for a microscopic investigation in which the basic ingredients for a proper description of such nanostructures are taken into account and might guide the experimental analysis as a kind of "reference spectrum" obtained under controlled geometrical conditions.

Here we present three such spectra obtained within timedependent local-spin density functional theory (TDLSDFT). The first two correspond to circularly symmetric nanorings made of 12 and 22 electrons embedded into a GaAs$\mathrm{Ga}_{x} \mathrm{Al}_{1-x} \mathrm{As}$ heterostructure. Although the method can handle a smaller number of electrons, the possibility of describing the two-electron structure ${ }^{18}$ is beyond its reach, and for that reason we have limited it from the start, presenting only results obtained for a five-electron nanoring of equal size as a third example. It is doubtless that the natural evolution of the field will make possible a quantitative compari- son between TDLSDFT and experimental results still to come.

This work is organized as follows. In Sec. II we discuss the results we have obtained for the ring ground states (gs's), which are the starting point for the study of their charge density excitations presented in Sec. III. Finally, concluding remarks are given in Sec. IV.

\section{GROUND STATE OF QUANTUM RINGS}

We consider a circularly symmetric quantum ring made of $N$ electrons moving in the $z=0$ plane. The confining potential $V^{+}(r)$ is that created by $N^{+}$positive charges uniformly distributed between an outer $\left(R_{o}\right)$ radius and inner $\left(R_{i}\right)$ radius in the presence of a constant magnetic field $B$ in the positive $z$ direction. In the local-spin-density approximation (LSDA), the single-electron wave functions are given by the solution of the Kohn-Sham (KS) equation

$$
\begin{aligned}
{[-} & \frac{1}{2} \nabla^{2}+\frac{1}{2} \omega_{c} l_{z}+\frac{1}{8} \omega_{c}^{2} r^{2}-V^{+}(r)+V^{H}+V^{x c} \\
& \left.+\left(W^{x c}+\frac{1}{2} g^{*} \mu_{B} B\right) \sigma_{z}\right] \varphi_{\alpha}(r, \theta) \\
= & \epsilon_{\alpha} \varphi_{\alpha}(r, \theta) .
\end{aligned}
$$

The $V^{+}(r)$ potential is analytical and can be expressed in terms of the elliptic $\mathbf{E}$ and $\mathbf{K}$ functions: ${ }^{20}$

$$
V^{+}(r)=\frac{4 N^{+}}{\pi\left(R_{o}^{2}-R_{i}^{2}\right)}\left\{\begin{array}{l}
{\left[R_{o} \mathbf{E}\left(\frac{r}{R_{o}}\right)-R_{i} \mathbf{E}\left(\frac{r}{R_{i}}\right)\right] \text { if } r<R_{i}} \\
{\left[R_{o} \mathbf{E}\left(\frac{r}{R_{o}}\right)-r \mathbf{E}\left(\frac{R_{i}}{r}\right)\right]+r\left[1-\left(\frac{R_{i}}{r}\right)^{2}\right] \mathbf{K}\left(\frac{R_{i}}{r}\right) \text { if } R_{o}>r>R_{i}} \\
r\left\{\mathbf{E}\left(\frac{R_{o}}{r}\right)-\mathbf{E}\left(\frac{R_{i}}{r}\right)+\left[1-\left(\frac{R_{i}}{r}\right)^{2}\right] \mathbf{K}\left(\frac{R_{i}}{r}\right)-\left[1-\left(\frac{R_{o}}{r}\right)^{2}\right] \mathbf{K}\left(\frac{R_{o}}{r}\right)\right\} \text { if } r>R_{o} .
\end{array}\right.
$$

We have thoroughly used effective atomic units ( $\hbar$ $\left.=e^{2} / \epsilon=m=1\right)$, where $\epsilon$ is the dielectric constant of the semiconductor, and $m$ is the electron effective mass. The definition of the $V^{H}, V^{x c}$, and $W^{x c}$ potentials entering Eq. (1), as well as the values of the electron effective mass $\epsilon$ and $g^{*}$ we are using for GaAs, were given in the preceding paper. ${ }^{21}$

As previously indicated, we have considered two nanorings. The narrow one has $R_{o}=100 \mathrm{~nm}, R_{i}=70 \mathrm{~nm}, N$ $=12$, and $N^{+}=14$, and the broad one $R_{o}=100 \mathrm{~nm}, R_{i}$ $=37.5 \mathrm{~nm}, N=22$, and $N^{+}=24$. These values have been selected so that both rings have roughly the same average surface densities as in the $N=25$, quantum dot described in Refs. 22, 23, and 21, as well as the same outer radius. This would allow us to make a comparison between FIR modes arising in somehow similar dot and ring geometries. The radii ratio in the broad ring is similar to that of Ref. 18.

Figure 1 represents several electron densities for selected $B$ values as a function of the radial distance in the case of the
NR, and Fig. 2 in the case of the BR. In the latter case, at $B=0$ the central electron density is not zero, but it is around two orders of magnitude smaller than its maximum value. At present, it is unclear to us whether a different confining potential that prevents the electrons from having a sizable probability of being inside the ring "hole," like the parabolic confinement of Ref. 15, would be more realistic.

In the case of the NR, the electronic density has no structure, presenting a Gaussian-like shape whose width decreases with increasing $B$. In contradistinction, in the BR an incipient bulk density region appears as well as the characteristic "bump" at the edges clearly visible in dots confined by a disk geometry. ${ }^{23}$

Figures 3 and 4 represent the single-particle (sp) energies as a function of the single electron orbital angular momentum $l$ and different $B$ values. The $N=12$ ring becomes fully polarized between $B=2$ and $3 \mathrm{~T}$, and the $N=22$ ring between $B=3$ and $4 \mathrm{~T}$ (in the $N=25$ quantum $\operatorname{dot}^{23}$ it happens 


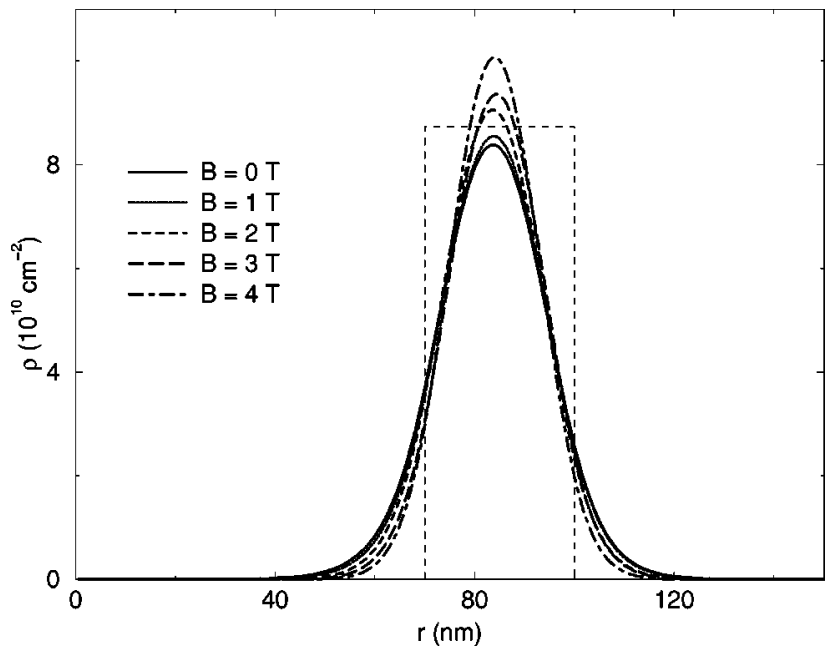

FIG. 1. Electronic densities $\left(10^{10} \mathrm{~cm}^{-2}\right)$ as a function of the radial distance $(\mathrm{nm})$ for the narrow ring.

at $B \sim 3.6 \mathrm{~T})$. It can be seen from the corresponding panels in these figures that at $B=0$ both rings have a $z$ component of the total spin different from zero, $S_{z}=1$. Since the $N=10$ and 20 rings are closed shell systems, this means that Hund's first rule is obeyed by these small rings, as it is in small dots. ${ }^{24}$

The sp energies are arranged into bands which are bent upwards at both ends not only at low $B$. This is a peculiarity of the ring geometry, which simultaneously bears the characteristics of dot and antidot bands, the former ones bending upwards at high $l$, and the later ones at small $l .^{25}$ The existence of two bendings when a magnetic field is applied is the microscopic origin of the two edge magnetoplasmons, as we shall discuss in the Sec. III.

When the ring becomes fully polarized, increasing $B$ further produces the displacement as a whole of the set of occupied sp levels to higher $l$ 's. We have found that this is the mechanism rings have to keep at its total orbital angular momentum $L_{z}$ increasing with $B$. That can be seen, for example, in the high- $B$ panels corresponding to the NR (see also Fig. 11). In Fig. 5 we plot the evolution of $L_{z}$ and $2 S_{z}$ with $B$ for the NR, and in Fig. 6 we do so for the BR.

The shifting upwards in $l$ of the whole sp spectrum with

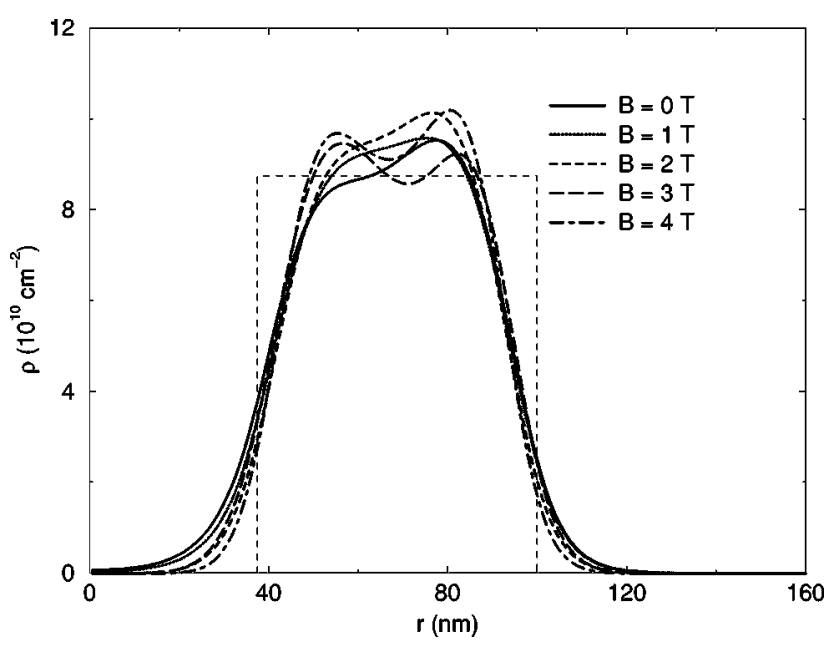

FIG. 2. Same as Fig. 1 for the broad ring.
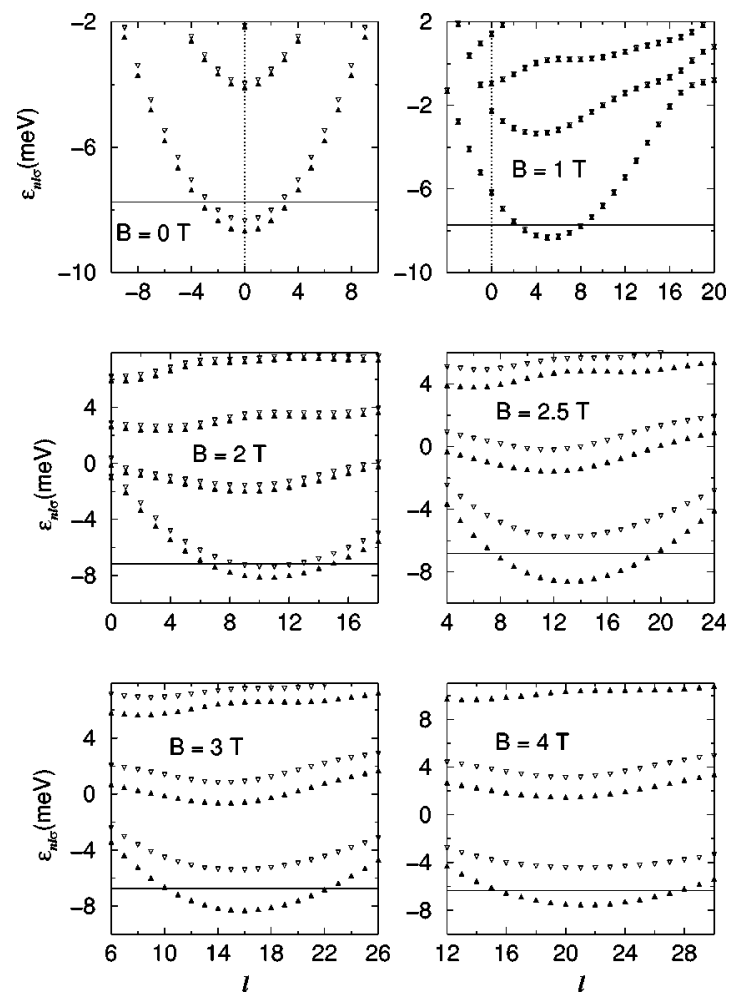

FIG. 3. Single-particle energies as a function of orbital angular momentum $l$ corresponding to the narrow ring. The horizontal lines represent the electron chemical potential. The full, upright triangles represent $\sigma=\uparrow$ bands, and the empty, downright triangles represent $\sigma=\downarrow$ bands.
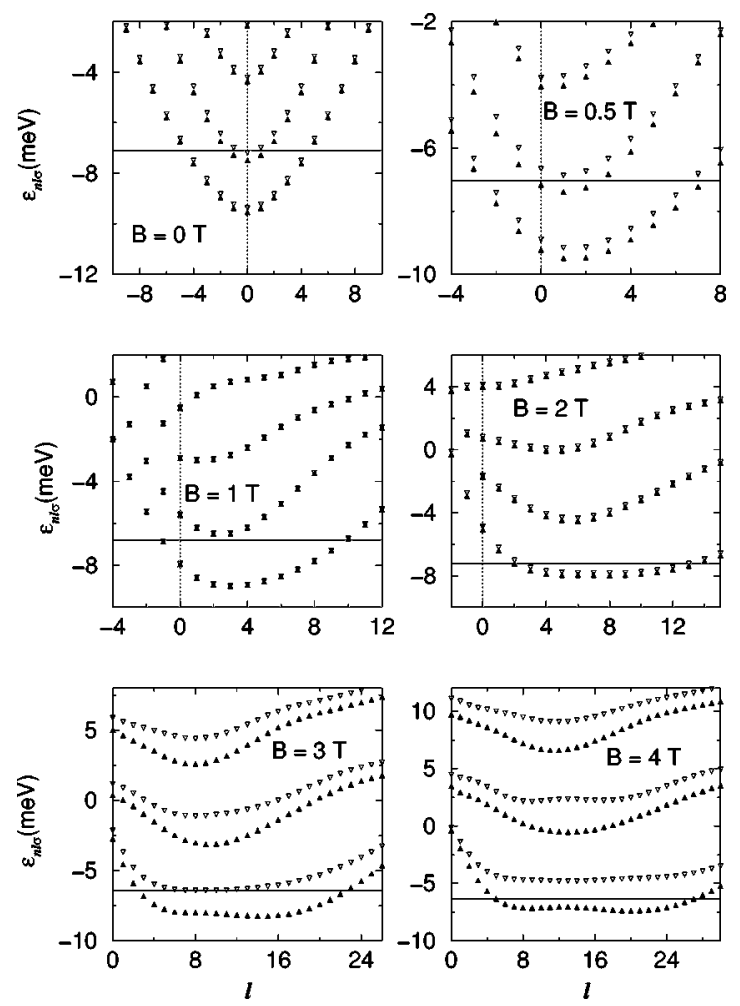

FIG. 4. Same as Fig. 3 for the broad ring. 


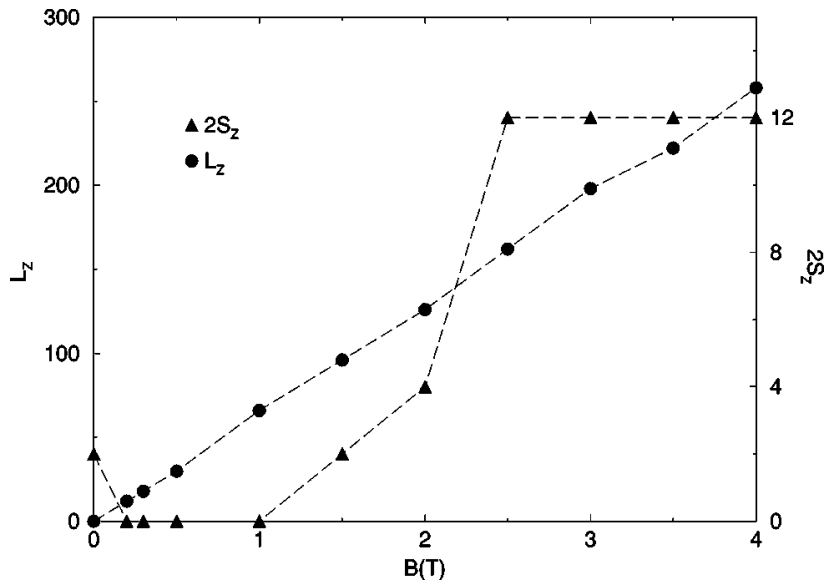

FIG. 5. Total orbital and spin angular momenta $L_{z}$ and $2 S_{z}$ as a function of $B$ for the narrow ring. The dashed lines are drawn to guide the eye.

increasing $B$ is a distinct characteristic of rings that deserves further investigation. In quantum dots, the stability region in the $N-B$ phase plane of the fully polarized configuration, called the maximum density droplet state, built from sp orbitals having $l=0,1,2 \ldots N-1^{26,27}$ is limited from the left by a line $B_{f}$ representing, for a given number number of electrons, the magnetic field at which $2 S_{z}=N$, and from the right by a line $B_{r}$ at which edge reconstruction starts. ${ }^{28-30}$ This is a rather narrow region, a few tenths of a tesla wide, ${ }^{30}$ because after being fully polarized the magnetic field is very effective in promoting electrons from high to higher $l \mathrm{sp}$ levels, reconstructing the dot edge. In rings this is not quite so, because the existence of an electron depletion at the center and the consequent upward bending of the sp bands allows for an alternative mechanism to keep increasing $L_{z}$ while retaining the simplicity of the gs wave function, namely, a Slater determinant made of the lowest possible $l \mathrm{sp}$ states from a minimum $l_{m}$ to a maximum $l_{M}$ such that $N$ $=l_{M}-l_{m}+1$. Taking as an example the situation of the NR, at $B=11 \mathrm{~T}$ we have found that $l_{m}=54$ and $l_{M}=65$. It might well happen that, for quantum rings, no equivalent kind of edge reconstruction mechanism exists, but addressing this point is beyond the capabilities of the density functional we are using.

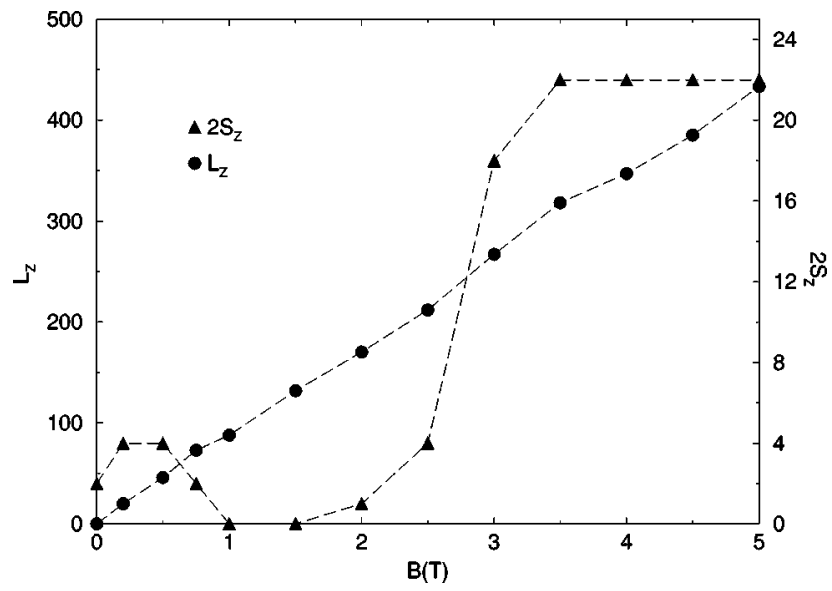

FIG. 6. Same as Fig. 5 for the broad ring.

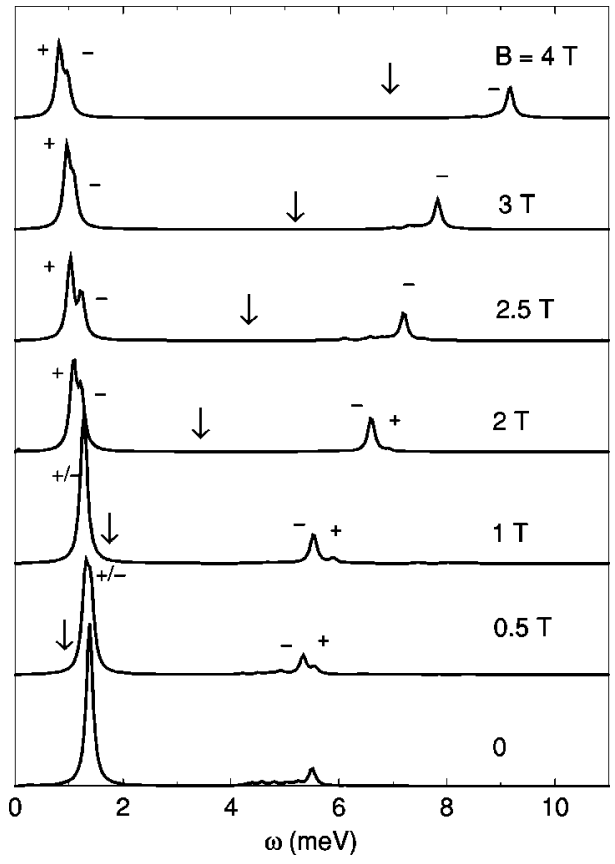

FIG. 7. Strength function (arbitrary units) as a function of the excitation energy $(\mathrm{meV})$ for the narrow ring at several $B$ values. The arrows indicate the value of the cyclotron frequency. The $(-)$ or $(+)$ symbol close to the more intense peaks denotes the character of the dipole polarization.

\section{CHARGE-DENSITY EXCITATIONS OF A QUANTUM RING}

Once the gs has been obtained, we determine the induced densities originating from an external field employing linearresponse theory. We refer the reader to Ref. 21 for a thorough discussion of the longitudinal response in quantum dots, and of its direct applicability to quantum rings.

Figures 7 and 8 show the charge-density strength function

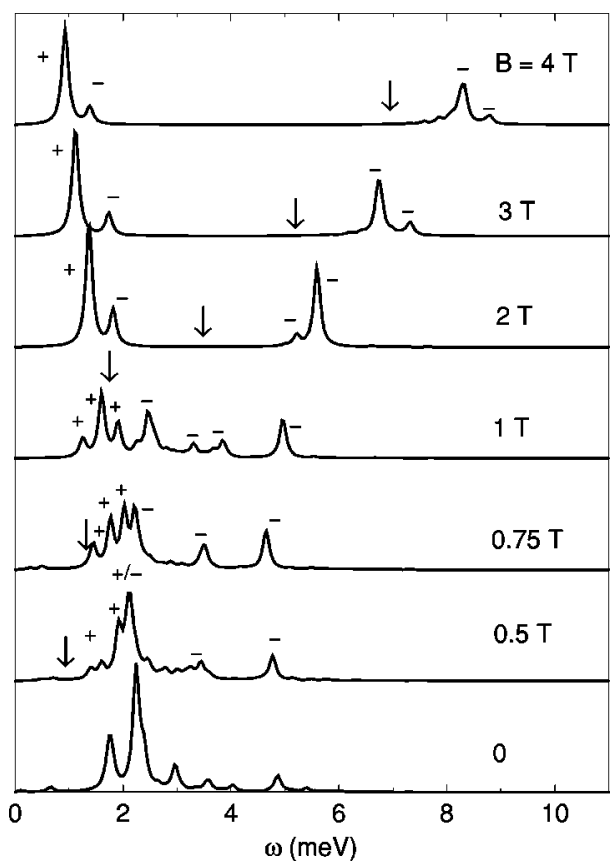

FIG. 8. Same as Fig. 7 for the broad ring. 
for the $N=12$ and 22 electron rings, respectively, and several $B$ values. The plus or minus sign close to the more intense peaks indicates that they originated either from $D_{\rho}^{(+1)}$ or $D_{\rho}^{(-1)}$, which are the dipole vectors defined in Ref. 21. Obviously, at zero magnetic field, $(+)$ and $(-)$ excitations are degenerated.

The CDE's displayed in these figures are easier to understand starting from the high- $B$ results, and keeping in mind the sp levels drawn in Figs. 3 and 4. First notice that as in dots, the $(+)$ low-energy modes are intraband CDE's from the outer ring boundary. The $(-)$ low-energy modes are intraband CDE's of the inner ring boundary, obviously absent in dots. However, they are the only edge modes in antidots. $^{3,4}$ The $(+)$ modes arise when the dipole field changes the total $L_{z}$ of the ring by +1 , and the $(-)$ modes when this change is -1 . Figures 3 and 4 show, indeed, that both kinds of edge modes are possible in rings.

The higher-energy peaks are bulk modes arising from interband transitions. At moderate- $B$ values, both positive and negative high-energy peaks are present in the strength, but at high- $B$ values only modes excited by $D_{\rho}^{(-1)}$ have an appreciable intensity: as in the dot case, the $(+)$ low-energy edge mode takes all the strength corresponding to the $D_{\rho}^{(+1)}$ operator.

Figure 3 shows that for some $B$ values, the sp energies are distributed following a very symmetric pattern as a function of $l$. This is the reason why sometimes $(+)$ and $(-)$ edge modes are nearly degenerated. Their splitting is not regular as a function of $B$, indicating a kind of "shell structure" effect that only a microscopic model can reveal. Still, the gross features of the three energy branches displayed in Fig. 7 is very similar to that of narrow microrings: ${ }^{9,11}$ two lowenergy edge modes with a negative $B$ dispersion, a highenergy mode which at low $B$ has a negative dispersion, and eventually a positive $B$ dispersion at high magnetic fields.

At zero magnetic field, or generally speaking, at low $B$, CDE's are delocalized as in the case of quantum dots. Notice, for instance, that $(+)$ and $(-)$ excitations are degenerated, and it makes no sense to associate either of them with excitations coming from the inner or outer ring boundary. In addition, in the case of BR's the low- $B$ strength is rather fragmented, rendering the analysis more complex. It is worth recalling that a similar fragmentation occurs in the case of dots if one uses a positively charged disk to model the confining potential. ${ }^{21}$ Still, two quite distinct structures, one at high excitation energies and another at low excitation energies, are present at $B=0$ in the case of rings, whose origin can be traced back from the results at high $B$, and one may associate the low-energy peaks with intraband transitions and the high-energy peaks with interband transitions.

In the BR case, the $B$ dispersion of the (-) edge mode is first positive; it reaches a maximum at around $B=1 \mathrm{~T}$, and then becomes negative. The high-energy peaks with appreciable strength are now only $(-)$ modes. Again, these features are those displayed by broad microrings.

For quantum dots and rings of similar size and electron number, one expects that the energy of the $B=0$ mode is lower for the ring than for the dot. Actually, this is an experimental fact ${ }^{18}$ that can be qualitatively explained using a sum rule method. We have found that at $B=0$ the average
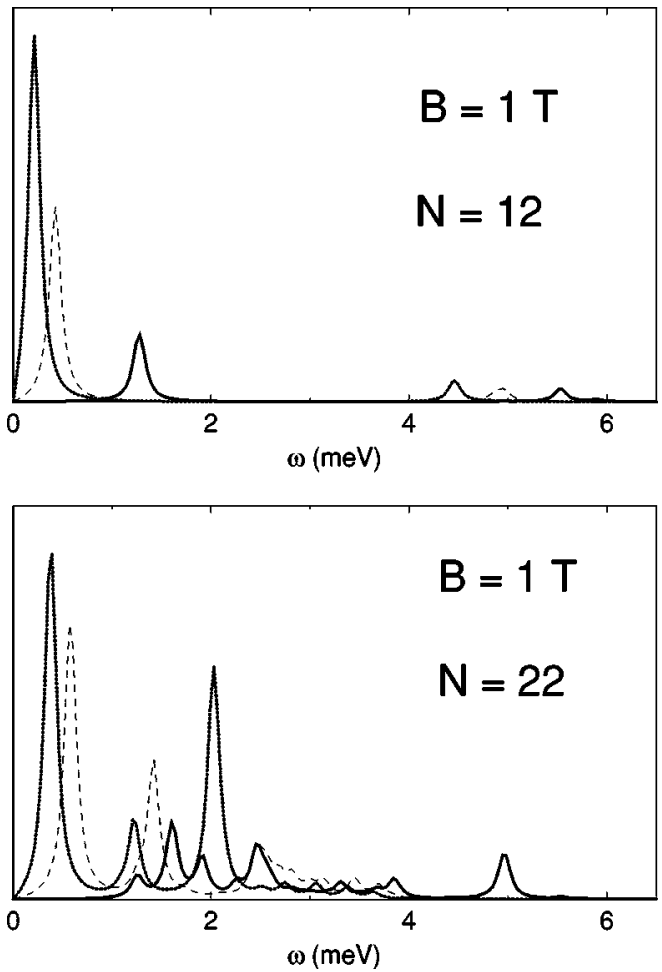

FIG. 9. Strength function (arbitrary units) at $B=1 \mathrm{~T}$ as a function of the excitation energy $(\mathrm{meV})$ for the narrow ring (top panel) and the broad ring (bottom panel). The solid line is the chargedensity response, the dotted line the longitudinal spin-density response, and the dashed line the free-electron response.

frequency of the dipole mode can be written as ${ }^{23}$

$$
\Omega^{2}=\frac{1}{2 N} \int d \vec{r} \Delta V^{+}(r) \rho(r) .
$$

Taking a parabola $\omega_{0}^{2} r^{2} / 2$ as the confining potential $V^{+}(r)$ for a dot, and $\omega_{0}^{2}(r-R)^{2} / 2$ for a ring of mean radius $R$ having the same number of electrons, one can easily check that $\Omega=\omega_{0}$ for the dot, and $\Omega \sim \omega_{0} / \sqrt{2}$ for a narrow ring, or for a ring broad enough so that the electronic density can be considered as being constant.

The FIR response of $\mathrm{BR}$ rings also has some features in common with antidots, which we recall that at $B \neq 0$ basically comes from the $D_{\rho}^{(-1)}$ component of the dipole operator. One is the $B$ dispersion of the inner edge mode. The other one is the transfer of strength from the low- to the high-energy ( - ) peak. ${ }^{3,4}$

In all cases we have studied, CDE's emerge as collective peaks. The residual electron-hole $(e-h)$ interaction shifts CDE's to higher energies from the sp excitations (SPE's) which constitute the free response (see Fig. 10 below). In the longitudinal spin case, the residual interaction is attractive but weak, as it is only due to the exchange-correlation potential. As an example, in Fig. 9 we show the three responses for the NR's and BR's at $B=1 \mathrm{~T}$.

Finally, we discuss the results we have obtained for a nanoring more similar to that experimentally studied. ${ }^{18} \mathrm{In}$ this case, $R_{o}=40 \mathrm{~nm}, R_{i}=15 \mathrm{~nm}$, and $N=N^{+}=5$. Figure 10 shows the charge-density strength function at several $B$ values, and Fig. 11 the sp energy levels. 


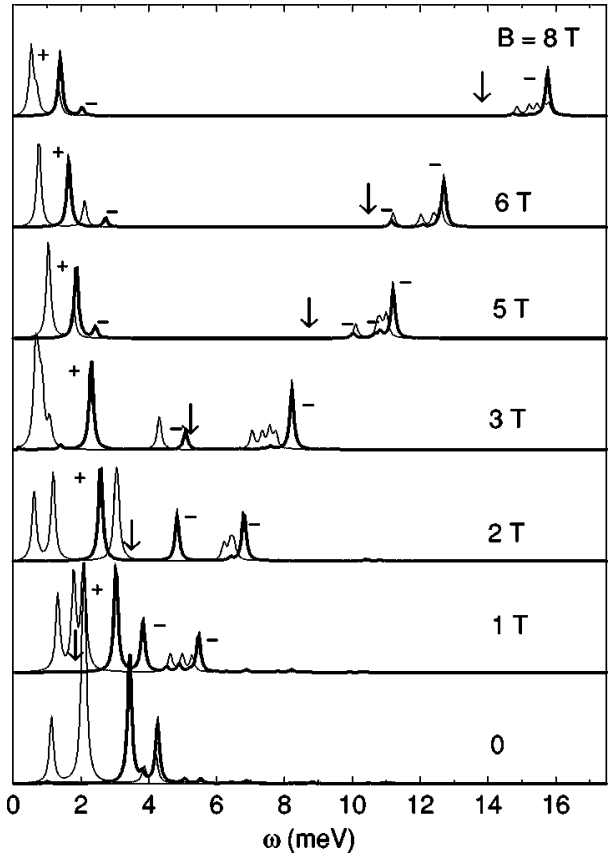

FIG. 10. Same as Fig. 7 for the $N=N^{+}=5$ nanoring with $R_{o}$ $=40 \mathrm{~nm}$ and $R_{i}=15 \mathrm{~nm}$. The free strength function is also plotted (thin lines).

Basically, the results are qualitatively similar to those of the broad nanoring already discussed (they have the same $R_{o} / R_{i}$ ratio). When a magnetic field is applied, one can clearly see the transfer of strength between the edge and bulk (-) branches as $B$ increases, which is quite similar to the
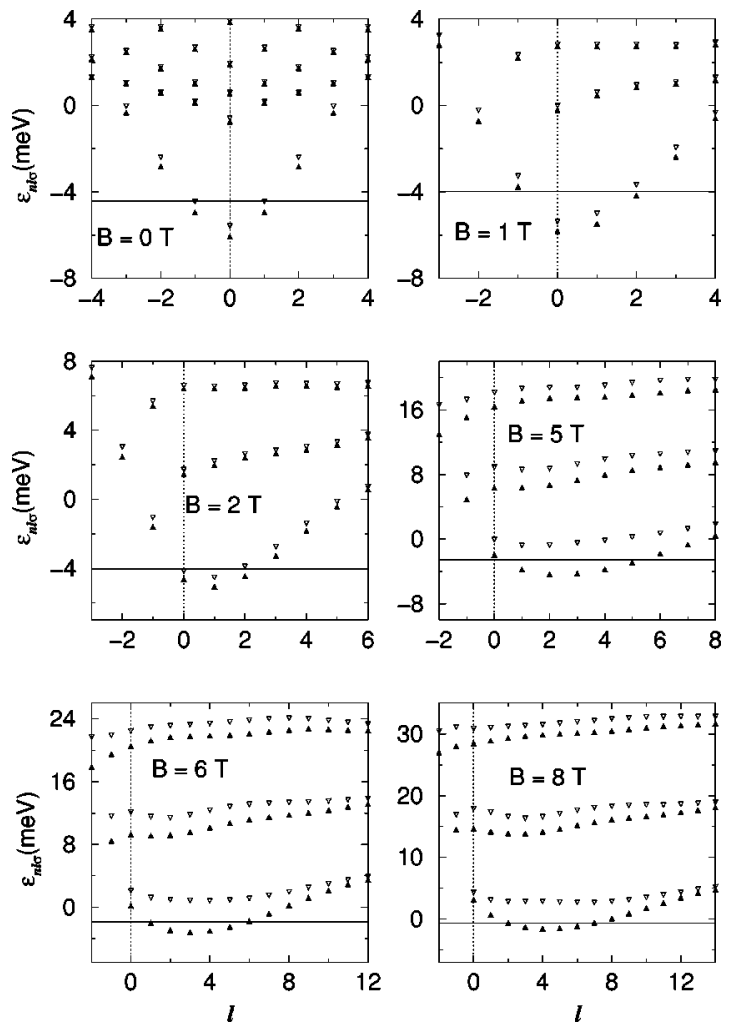

FIG. 11. Same as Fig. 3 for the $N=N^{+}=5$ nanoring, with $R_{o}$ $=40 \mathrm{~nm}$ and $R_{i}=15 \mathrm{~nm}$. antidot case already pointed out. The transfer is possible because both branches have the same polarization. The coupling is very inefficient in narrow rings, and the (-) highand low-energy peaks keep their own strength. This is the situation displayed in Fig. 7 for the $N=12$ ring.

It is worth noting the evolution of the $(-)$ edge mode with $B$, which is a rather high-energy mode with a positive $B$ dispersion from $B=1$ to $4 \mathrm{~T}$, and whose energy abruptly falls between 4.5 and $5 \mathrm{~T}$. This decrease is due to a change in the occupied sp levels, which illustrates the relevance of shell effects especially in the case of a small number of electrons. A look at the panels corresponding to $B=2$ and $5 \mathrm{~T}$ in Fig. 11 explains the effect. It can be seen how asymmetrically are distributed the sp levels, with a much larger energy difference for the $e-h$ pairs contributing to the edge excitation of the inner ring boundary than for those building the edge excitation of the outer boundary. This explains the large energy of the (-) edge excitation up to $B \sim 4.5 \mathrm{~T}$. Of course, this is a qualitative argument, since the residual $e-h$ interaction has a sizable effect in the charge-density channel. Conversely, at $B=5 \mathrm{~T}$ and above, the sp levels are distributed more symmetrically, the $e$ - $h$ energy differences are smaller, and the $(+)$ and $(-)$ edge modes follow the BR systematics.

\section{SUMMARY AND OUTLOOK}

In this work we have studied CDE's in quantum rings in some detail. We have confirmed the expectations put forward by Dahl et al. ${ }^{9}$ that plasmon resonances in quantum rings are dominated to a large extent by geometric effects, although shell effects may cause, in the case of few electron nanorings, effects that cannot be systematized. Apart from an example, we have restricted our analysis to CDE's. It would be as simple to describe SDE's and SPE's within TDLSDFT, much along the case of quantum dots, ${ }^{21}$ if experimental information becomes available.

Our work complements the theoretical description of microrings made by Zaremba. ${ }^{11}$ Even if a kind of characteristic pattern can be established for narrow or broad nanorings, this confining geometry allows us to study much richer spectra than in dots or antidots. It might then offer the possibility of testing theoretical descriptions that equally well describe plasmon modes in quantum dots, even if their complexity is quite different.

The lack of experimental results for nanorings hosting several electrons has not allowed us to make a quantitative comparison of our calculations with experiments. A qualitative comparison between the calculated $N=5$ spectrum and the measured $N=2$ FIR spectrum ${ }^{18}$ is inconclusive. To arrange the peaks into branches and disentangle the $B$ dispersion of the plasmon modes unambiguously, it would be essential to assign the polarization state experimentally to the main energy peaks. This has been paramount in the analysis of the theoretical FIR response, which otherwise would have not allowed us to distinguish between peak fragmentation and different plasmon branches in some cases. Alternatively, calculations for rings with as many electrons as in the experiments might guide one to distribute the experimental data into branches. TDLSDFT may be a useful tool for doing 
this in nanorings with a few more electrons than those studied so far. Other, more microscopic, methods ${ }^{14}$ are better suited for a two-electron ring provided the geometry is adjusted to the experimental situation. Finally, we have also determined that Hund's first rule is fulfilled in the quantum rings we have studied, and have elucidated a possible mechanism by which a fully polarized quantum ring may have a rather simple gs structure in a wide range of magnetic fields.

\section{ACKNOWLEDGMENTS}

We are most indebted to Dr. Axel Lorke for a very useful correspondence. This work was performed under Grant Nos. PB95-1249 and PB95-0492 from DGICYT, and 1998SGR00011 from Generalitat de Catalunya. A.E. and M.B. (Ref. PR1997-0174) acknowledge support from the DGES (Spain).
*Permanent address: Departament d'Estructura i Constituents de la Matèria, Facultat de Física, Universitat de Barcelona, E-08028 Barcelona, Spain.

${ }^{1}$ L. Jacak, P. Hawrylak, and A. Wójs, Quantum Dots (Springer, Berlin, 1998).

${ }^{2}$ Mesoscopic Physics and Electronics, edited by T. Ando, Y. Arakawa, K. Furuta, S. Komiyama, and H. Nakashima (Springer, Berlin, 1998)

${ }^{3}$ K. Kern, D. Heitmann, P. Grambow, Y. H. Zhang, and K. Ploog, Phys. Rev. Lett. 66, 1618 (1991)

${ }^{4}$ Y. Zhao, D. C. Tsui, M. Santos, M. Shayegan, R. A. Ghanbari, D. A. Antoniadis, and H. I. Smith, Appl. Phys. Lett. 60, 1510 (1992)

${ }^{5}$ A. Lorke, I. Jejina, and J. P. Kotthaus, Phys. Rev. B 46, 12845 (1992).

${ }^{6}$ G. Y. Wu and Y. Zhao, Phys. Rev. Lett. 71, 2114 (1993).

${ }^{7}$ S. A. Mikhailov, Phys. Rev. B 54, R14 293 (1996).

${ }^{8}$ A. Emperador, M. Pi, M. Barranco, E. Lipparini, and Ll. Serra, Phys. Rev. B 58, 6732 (1998).

${ }^{9}$ C. Dahl, J. P. Kotthaus, H. Nickel, and W. Schlapp, Phys. Rev. B 48, 15480 (1993)

${ }^{10}$ C. R. Proetto, Phys. Rev. B 46, 16174 (1992).

${ }^{11}$ E. Zaremba, Phys. Rev. B 53, R10 512 (1996).

${ }^{12}$ D. Huang and G. Gumbs, Phys. Rev. B 46, 4147 (1992).

${ }^{13}$ V. Gudmundsson and Á. Loftsdóttir, Phys. Rev. B 50, 17433 (1994).

${ }^{14}$ L. Wendler, V. M. Fomin, A. V. Chaplik, and A. O. Govorov, Phys. Rev. B 54, 4794 (1996).

${ }^{15}$ T. Chakraborthy and P. Pietiläinen, Phys. Rev. B 50, 8460 (1994).
${ }^{16}$ D. Mailly, C. Chapelier, and A. Benoit, Phys. Rev. Lett. 70, 2020 (1993).

${ }^{17}$ J. M. Garcia, G. Mediros-Ribeiro, K. Schmidt, T. Ngo, J. L. Feng, A. Lorke, J. Kotthaus, and P. M. Petroff, Appl. Phys. Lett. 71, 2014 (1997).

${ }^{18}$ A. Lorke and R. J. Luyken, Physica B 256, 424 (1998).

${ }^{19}$ A. Kumar, S. E. Laux, and F. Stern, Phys. Rev. B 42, 5166 (1990).

${ }^{20}$ I. S. Gradshteyn and I. M. Ryzhik, Table of Integrals, Series and Products (Academic, New York, 1980).

${ }^{21}$ L1. Serra, M. Barranco, A. Emperador, M. Pi, and E. Lipparini, preceding paper, Phys. Rev. B 59, 15290 (1999).

${ }^{22}$ T. Demel, D. Heitmann, P. Grambow, and K. Ploog, Phys. Rev. Lett. 64, 788 (1990).

${ }^{23}$ E. Lipparini, N. Barberan, M. Barranco, M. Pi, and Ll. Serra, Phys. Rev. B 56, 12375 (1997).

${ }^{24}$ S. Tarucha, D. G. Austing, T. Honda, R. J. van der Hage, and L. P. Kouwenhoven, Phys. Rev. Lett. 77, 3613 (1996).

${ }^{25}$ A. Emperador, M. Pi, M. Barranco, E. Lipparini, and Ll. Serra (unpublished).

${ }^{26}$ A. H. MacDonald, S.-R. Eric Yang, and M. D. Johnson, Aust. J. Phys. 46, 345 (1993).

${ }^{27}$ C. de C. Chamon and X.-G. Wen, Phys. Rev. B 49, 8227 (1994).

${ }^{28}$ S.-R. Eric Yang, A. H. MacDonald, and M. D. Johnson, Phys. Rev. Lett. 71, 3194 (1993).

${ }^{29}$ O. Klein, C. de C. Chamon, D. Tang, D. M. Abusch-Magder, U. Meirav, X.-G. Wen, M. A. Kastner, and S. J. Wind, Phys. Rev. Lett. 74, 785 (1995).

${ }^{30}$ M. Ferconi and G. Vignale, Phys. Rev. B 56, 12108 (1997). 